\title{
Pharmacological inhibition of poly (ADP-ribose) polymerase by olaparib ameliorates influenza-virus-induced pneumonia in mice
}

\author{
Wei Liu ${ }^{1} \cdot$ Xiaojuan Ren $^{2} \cdot$ Qian Wang ${ }^{2} \cdot$ Yan Zhang ${ }^{2} \cdot$ Junfeng Du ${ }^{3}$
}

Received: 1 July 2020 / Accepted: 24 August 2020 / Published online: 31 August 2020

(C) Springer-Verlag GmbH Germany, part of Springer Nature 2020

\begin{abstract}
Treatments against influenza A viruses (IAV) have to be updated regularly due to antigenic drift and drug resistance. Poly (ADPribose) polymerases (PARPs) are considered effective therapeutic targets of acute lung inflammatory injury. This study aimed to explore the effects of PARP-1 inhibitor olaparib on IAV-induced lung injury and the underlying mechanisms. Male wild-type C57BL/6 mice were intranasally infected with IAV strain H1N1 to mimic pneumonia experimentally. Olaparib at different doses was intraperitoneally injected 2 days before and 5 consecutive days after virus stimulation. On day 6 post-infection, lung tissues as well as bronchoalveolar lavage fluid (BALF) were sampled for histological and biochemical analyses. Olaparib increased the survival rate of IAV mice dose-dependently. Olaparib remarkably reduced IAV mRNA expression, myeloperoxidase (MPO) level, and inflammatory cell infiltration in IAV lungs. Moreover, olaparib significantly reduced the level of interleukin (IL)-1 $\beta$, tumor necrosis factor (TNF)- $\alpha$, interferon (IFN)- $\gamma$, IL-6, and IL-4 and increased IL-10 in IAV lungs. Also, olaparib efficiently reduced IL-6, monocyte chemotactic protein (MCP)-1, granulocyte colony-stimulating factor (G-CSF), TNF- $\alpha$, chemokine (C$\mathrm{X}-\mathrm{C}$ motif) ligand (CXCL)1, CXCL10, chemokine (C-C motif) ligand (CCL)3, and regulated on activation, normal $\mathrm{T}$ cell expressed and secreted (RANTES) release in IAV BALF. Olaparib decreased PARylated protein content and p65, IkB $\alpha$ phosphorylation in IAV lung tissues. This study successfully constructed the pneumonia murine model using IAV. Olaparib decreased IAV-induced mortality in mice, lung injury, and cytokine production possibly via modulation of PARP-1/NF- $\mathrm{BB}$ axis.
\end{abstract}

Keywords Influenza A virus · PARP · Olaparib · Pneumonia · Cytokines

\section{Introduction}

Influenza is an acute infectious disease affecting respiratory tracts accompanied with different clinical manifestations ranging from wild to lethal. Influenza causes seasonal, unpredictable

Electronic supplementary material The online version of this article (https://doi.org/10.1007/s10096-020-04020-5) contains supplementary material, which is available to authorized users.

\section{Wei Liu}

liuwei19801116@163.com

1 Department of Clinical Laboratory, Cangzhou Central Hospital, No. 16 Xinhua West Road, Cangzhou 061001, Hebei, China

2 Department of Infectious Diseases Medicine, Cangzhou Central Hospital, No. 16 Xinhua West Road, Cangzhou 061001, Hebei, China

3 Department of Respiratory and Critical Care Medicine, Cangzhou Central Hospital, No. 16 Xinhua West Road,

Cangzhou 061001, Hebei, China epidemics and it is now one of the major public health concerns worldwide [1, 2]. According to the report of the World Health Organization (WHO) posted online in 2018, only influenza A virus (IAV) has caused pandemics up to now, and most human influenza cases are due to the infection of two IAV strains, H1N1 and H3N2. IAV have laid heavy burdens on global population and economy these years [3]. Although vaccine inoculation and antiviral drug administration have been proved to be effective ways to control influenza, epidemics occur sometimes as a result of antigenic drift, which urges the development of novel anti-influenza drugs [4].

Poly (ADP-ribose) polymerases (PARPs) family is composed of 18 members which are involved in the bioprocesses, including DNA repair, cell cycle regulation, transcription, and so on $[5,6]$. Abnormal expression of PARPs is correlated with necrotic cell death, cancer, and some inflammatory disorders [7]. PARP-1 activation has been regarded as one of the critic mechanisms underlying lung inflammation in the context of lipopolysaccharide (LPS) and elastase stimulations experimentally $[8,9]$. Evidences indicated the abnormal increased 
expression of PARP-1 in non-pulmonary cells, alveolar epithelial cells, and lung tissue after IAV infection [10, 11], suggesting its potential as the target for the treatment of IAV infection. One of the PARP inhibitors, olaparib, is reported to ameliorate acute lung injury induced by elastase and LPS $[9,12]$. Yet the role of olaparib on IAV-induced lung injury has rarely been reported. This study aimed to explore the effects of PARP inhibition by olaparib on IAV infection.

\section{Methods and materials}

\section{Animals}

Specific-pathogen-free 8- to 9-week-old male wild-type (WT) C57BL/6 mice, weighing 25 to $30 \mathrm{~g}$, were purchased from the Nanjing Model Animal Center and kept at $25^{\circ} \mathrm{C}$ in a 12-h light/ dark cycle, with free access to food and water. Animals were allowed to acclimate to the housing environment for 1 week before experimental procedures. All the animal-involved experiments were performed in accordance with the Animal Care and Use Committee of Cangzhou Central Hospital.

\section{Influenza virus}

The A/Font Monmouth/47(H1N1, FM1) mouse-adapted influenza virus (Chinese Center for Disease Control and Prevention) was first plaque purified in the Madin-Darby canine kidney MDCK cells, followed by replication in 9-day-old chicken embryos. The virus pool was pretitrated in mice before further studies in order to determine a suitable challenge dose.

\section{Modeling of viral pneumonia in mice}

The murine model of viral pneumonia was constructed by intranasal infection with H1N1 according to previously described [13]. Briefly, ketamine (50 mg/kg weight) and pentobarbital (30 mg/kg weight) were intraperitoneally injected to induce the mild anesthesia in mice. Next, a $50-\mu l$ influenza virus in ice-cooled PBS, containing fifteen 50\% mouse lethal challenge doses (MLD50), was infected intranasally. Mice were kept on a $37{ }^{\circ} \mathrm{C}$ thermal insulation blanket for $20 \mathrm{~min}$ to recover. The day of virus infection was defined as experimental day 1 and 0-days post-infection.

\section{Grouping and drug treatment}

Two grouping methods were applied in this study. For the part of survival rate analysis, mice were randomly divided into 5 groups, that is, the influenza A virus (IAV) group (H1N1 virus + normal saline), the Ola groups (H1N1 virus $+2.5,5$, $10 \mathrm{mg} / \mathrm{kg}$ olaparib), and the positive control group (H1N1 virus $+10 \mathrm{mg} / \mathrm{kg}$ oseltamivir). For the part of biochemical detection, mice were divided into 3 groups, the control group (normal saline), the IAV group (H1N1 virus + normal saline), and the IAV + Ola group (H1N1 virus $+10 \mathrm{mg} / \mathrm{kg}$ olaparib).

Olaparib (Selleck Chemicals, Houston, TX, USA) at different doses was intraperitoneally injected 2 days before and 5 consecutive days after $\mathrm{H} 1 \mathrm{~N} 1$ virus challenge. The day of virus challenge was defined as experimental day 1. Oseltamivir (Hoffmann-La Roche, Basel, Switzerland) was administered in a similar manner to olaparib.

\section{Survival analysis}

After anesthetization, mice were infected intranasally with $50-\mu$ influenza virus in ice-cooled PBS, containing five MLD50, at experimental day 1 (0 days post-infection). Then mice in different groups were followed for 15 consecutive days post-infection with the number of deaths being monitored daily.

\section{Lung indexing}

The severity of pulmonary edema induced by H1N1 virus challenge was quantified by lung indexing. Five days after virus infection, the body weight of mice was measured using an electronic analytical balance. Then lungs were dissected, washed in pre-cooled PBS, and weighed. The lung index was calculated by the following formula: lung weight/body weight $\times 100 \%$.

\section{Lung histopathological grading}

At day 6 post-infection, lungs were dissected and fixed in $4 \%$ paraformaldehyde for further histopathological analysis. Paraffin sections in 5- $\mu \mathrm{m}$ thickness were stained with hematoxylin and eosin (H\&E) and examined using a light microscope. The severity of the lung injury was assessed by scoring the H1N1-induced lung histopathological changes using the scoring system previously described [14]. In brief, the grading was conducted in a blinded manner with the grader unaware of the concrete group or mice being reviewed. The scores of 0 to 4 were defined to represent normal, mild, severe, and very severe lung injury, respectively. In concrete, 0 was valued for normal lung, 1 for lower than $25 \%, 2$ for $25-50 \%, 3$ for $50-75 \%$, and 4 for higher than $75 \%$ lung involvement, respectively.

\section{Lung homogenates and bronchoalveolar lavage fluid (BALF) sampling}

At day 6 post-infection, lung homogenates and BALF were sampled from different groups of mice. For lung homogenates, the lungs were dissected and homogenized in ice-cold RIPA lysis (Sigma) supplemented with protease inhibitor (Sigma) on ice for $2 \mathrm{~h}$. After $4{ }^{\circ} \mathrm{C}$ centrifugations at $4000 \mathrm{~g}$ 
for $20 \mathrm{~min}$, supernatants were collected and stored at $-80^{\circ} \mathrm{C}$ for further biochemical analysis, including myeloperoxidase (MPO), cytokines, and targeted protein levels. Total protein content of lung homogenates was determined using a BCA assay kit (Beyotime, Shanghai, China). For BALF sampling, bronchoalveolar lavage was conducted according to previously described by Yashiro $\mathrm{M}$ et al. [15]. Briefly, after anesthesia, the left main bronchus was ligated and the right lung was quickly lavaged twice by $1 \mathrm{ml}$ of cold sterile PBS. Then the collected BALF was centrifuged at $2000 \mathrm{rpm}$ for $10 \mathrm{~min}$ at $4{ }^{\circ} \mathrm{C}$, and the supernatant was stored at $-80^{\circ} \mathrm{C}$ for measurement of cytokine levels.

\section{MPO activity detection}

MPO activity was assessed by 3,3',5,5'-Tetramethylbenzidine (TMB, Sigma-Aldrich, St. Louis, MO, USA) method according to previously described [16]. In brief, equal amount of sample $(10 \mu \mathrm{l})$ was sequentially mixed with $0.75-\mathrm{mM}$ hydrogen peroxide $(80 \mu \mathrm{l}), 2.9-\mathrm{mM}$ TMB dissolved in $14.5 \%$ dimethyl sulfoxide $(110 \mu \mathrm{l})$, and $150-\mathrm{mM}$ sodium phosphate buffer ( $\mathrm{pH} 5.4$ ) in a 96-well plate for 5-min incubation at $37{ }^{\circ} \mathrm{C}$. Then 2-M sulfuric acid (Sigma) was added to stop the reaction and absorbance at $450 \mathrm{~nm}$ was measured to evaluate MPO activity in each sample. The MPO levels were expressed as pmol MPO per milligram of lung tissue ( $\mathrm{pmol} / \mathrm{mg}$ protein).

\section{Determination of cytokines and chemokines}

Cytokines and chemokines in lung homogenate and BALF samples were measured using commercial assay kits according to the recommended protocols. Interleukin-1 $\beta$ (IL-1 $\beta$ ), interleukin-4 (IL-4), interleukin-6 (IL-6), interleukin-10 (IL-10), interferon- $\gamma$ (IFN- $\gamma$ ), and tumor necrosis factor- $\alpha(\mathrm{TNF}-\alpha)$ were measured by enzyme-linked immunosorbent assay (ELISA) kits (R\&D system, Minneapolis, MN, USA). Monocyte chemotactic protein-1 (MCP-1), granulocyte colony-stimulating factor $(\mathrm{G}-$ $\mathrm{CSF}$ ), chemokine ( $\mathrm{C}-\mathrm{X}-\mathrm{C}$ motif) ligand 1 (CXCL1), chemokine (C-X-C motif) ligand 10 (CXCL10), chemokine (C-C motif) ligand 3 (CCL3), and regulated on activation, normal $\mathrm{T}$ cell expressed and secreted (RANTES) were measured using a mouse multi-cytokine/chemokine magnetic bead panel (Millipore, Billerica, MA, USA) and analyzed on a Luminex 100 system (Luminex, Austin, TX, USA). The level of target factors was expressed as $\mathrm{pg} / \mathrm{mg}$ protein for lung homogenate detection and $\mathrm{pg} / \mathrm{ml}$ for BALF detection.

\section{Real-time quantitative polymerase chain reaction (RT- qPCR)}

Viral load was determined by RT-qPCR according to previously described by Li Y et al. [17] with slight modifications.
The total RNA of isolated lung tissues at day 6 post-infection was extracted using TRIzol method (Life technologies, Carlsbad, CA, USA) according to the manufacturer's instructions. Then equal amount of RNA for each sample was transcribed into complementary DNA (cDNA) using the First Strand cDNA synthesis kit (TaKaRa, Dalian, China) following the instructions. Quantitative PCR (qPCR) was performed using a SYBR Green suit (TaKaRa) to determine the mRNA expression levels of target genes, IAV M gene, and GAPDH, in an ABI real-time PCR system (Applied Biosystems, New York, NY, USA), with the following amplification procedures: 1-min preincubation at $98^{\circ} \mathrm{C}, 40$ cycles of $98^{\circ} \mathrm{C}$ for $10 \mathrm{~s}, 56{ }^{\circ} \mathrm{C}$ for $20 \mathrm{~s}$, and $72{ }^{\circ} \mathrm{C}$ for $30 \mathrm{~s}$. Each sample was performed in triplicate in one single technical repetition. The primer sequences used in this study were as follows: IAV M, 5'-AATGGTGCAGGCGATAGAG-3' (forward) and 5'TACTTGCGGCAACAACGAGAG-3' (reverse); GAPDH, 5'-TGAGGTCAATGAAGGGGTCG-3' (forward) and 5'TGAGGTCAATGAAGGGGTCG-3' (reverse). The relative quantitation of IAV was calculated using the comparative $2^{-\Delta \Delta \mathrm{Ct}}$ method. GAPDH was used as the inner control.

\section{Western blot analysis}

The PARylated protein content in the lung tissues was analyzed by western blot. Briefly, $-80{ }^{\circ} \mathrm{C}$ stored lung homogenate samples were thawed on ice. For each sample, $25-\mu \mathrm{g}$ protein was separated through $10 \%$ sodium dodecyl sulfatepolyacrylamide gel electrophoresis and transferred to a polyvinylidene difluoride membrane (Millipore), followed by 1 -h block in $5 \%$ nonfat milk at room temperature. Then the membranes were incubated at $4{ }^{\circ} \mathrm{C}$ overnight with primary antibodies against PAR (1:1000, Abcam, Cambridge, UK), phospho-p65 (p-p65, 1:2000, Abcam), p65 (1:2000, Abcam), phospho-IкB $\alpha$ (p-IкB $\alpha, 1: 1500$, Abcam), IкB $\alpha$ (1:1500, Abcam), or $\beta$-actin (1:2000, Santa Cruz, CA, USA), respectively. After the 2 -h incubation with peroxidase-conjugated secondary antibodies (Abcam) at room temperature, the enhanced chemiluminescence kit (Millipore) was used to detect the proteins of interest in the UVP BioSpectrum Imaging system (BioSpectrum, CA, USA). $\beta$ actin served as the inner control.

\section{Statistical analysis}

Each experiment was performed for at least three times. Data were expressed as mean \pm SD for each assay. Statistical analysis was conducted by a one-way analysis of variance (ANOVA) test using the SPSS software (Chicago, IL, USA). $p<0.05$ was considered to be statistically significant. For the body weight change analysis, the two-way ANOVA followed by Tukey's multiple comparison test was used. 

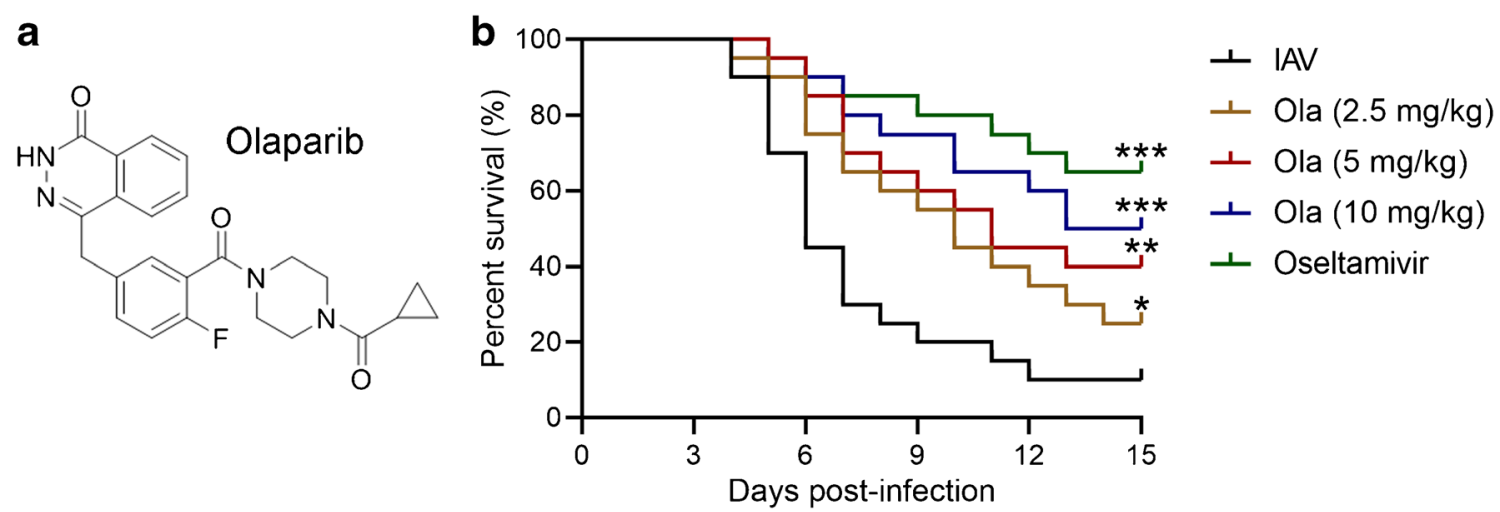

Fig. 1 Effects of olaparib on influenza virus challenge-induced mortality in mice. (a) Chemical structure of olaparib. (b) Mice from each experimental group were treated for 2 days before viral challenge and 6 days after infection and survival for 15 consecutive days. Oseltamivir

was used as positive control. Ola, olaparib group; IAV, influenza A virusinfected group. $n=20$ in each group. ${ }^{*} p<0.05,{ }^{* *} p<0.01$, and *** $p<0.001$ compared with the IAV infection group

\section{Results}

\section{Olaparib decreased IAV-induced mortality in mice}

This study aimed to investigate whether olaparib (chemical structure in Fig. 1a) possessed protective effects against influenza virus challenge. From day 3 post-infection, the IAVinfected mice began to exhibit clinical symptoms, and on day 6 post-infection, the symptoms got worse, which included but not limited to the following, such as weight loss (Fig. S1),

inactivity, rapid shallow breathing, and poor appetite, indicating the successful construction of viral pneumonia model experimentally. Figure $1 \mathrm{~b}$ showed that the IAV-only mice began to die on day 4 post-infection until day 12 post-infection. The positive control, oseltamivir, improved the survival rate of infected mice significantly compared with those in the IAVonly group. To the expectations, mice in the olaparib group showed higher survival rate compared with that in the IAV group in a dose-dependent manner, indicating that olaparib could powerfully protect against influenza virus challenge in
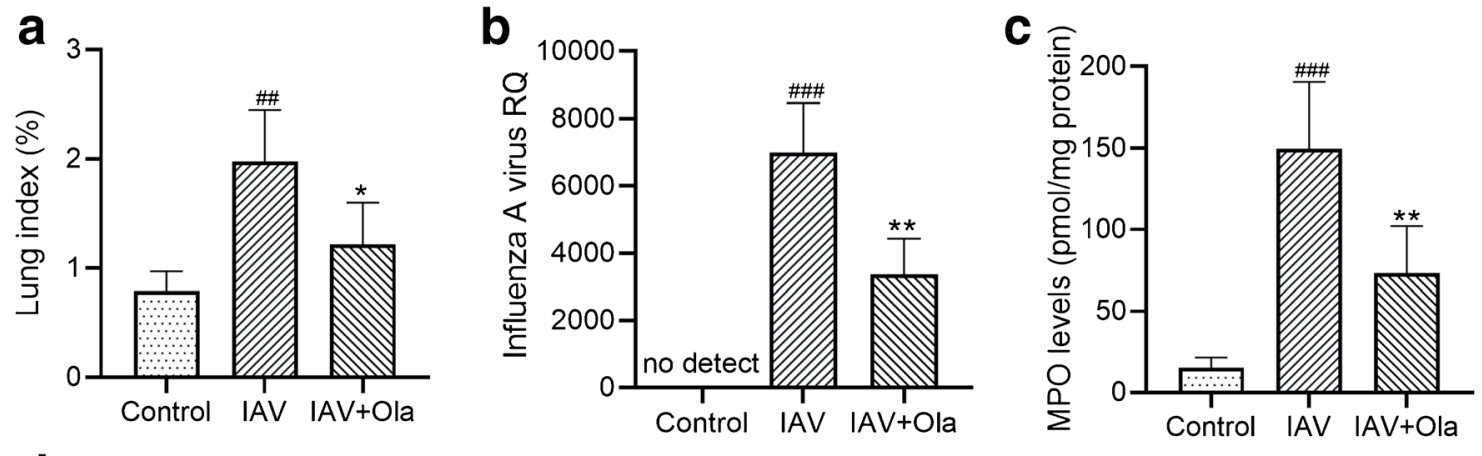

d
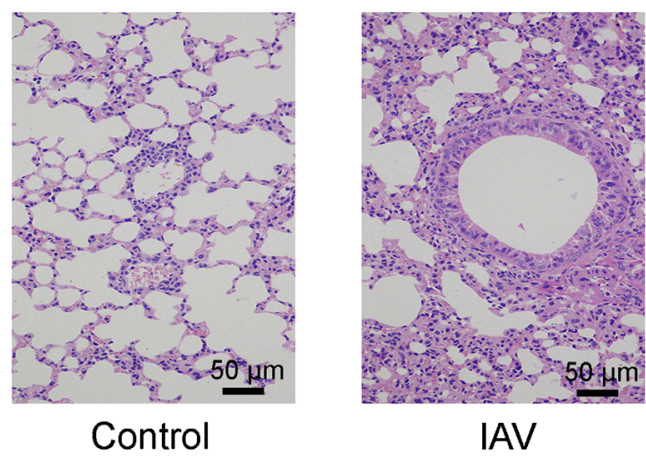

Fig. 2 Effects of olaparib on influenza virus challenge-induced lung injuries at day 6 post-infection. (a) Lung index ( $n=5$ for each group). (b) Relative quantitation of influenza A virus in lung tissues ( $n=8$ for each group). (c) MPO levels, a marker for leukocyte infiltration in lung tissues ( $n=8$ for each group). (d) Morphological changes were evaluated

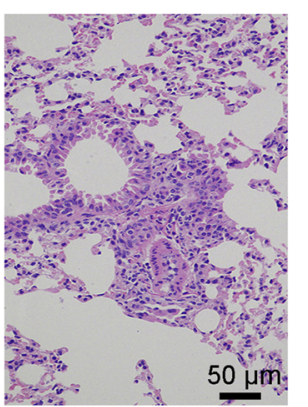

e

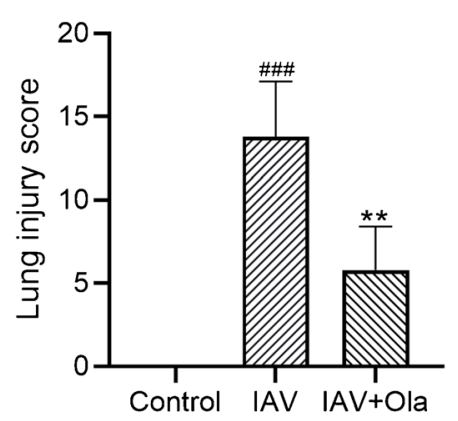

by $H \& E$ staining and the quantitative analysis of histological changes in the lung tissues (e) ( $n=8$ for each group). Data were presented as mean \pm SD. ${ }^{\# \#} p<0.01$ and ${ }^{\# \# \#} p<0.001$ compared with the control. ${ }^{*} p<0.05$ and $p<0.01$ compared with IAV 
mice. Additionally, olaparib-treated IAV mice exhibited lower weight loss compared with untreated ones, suggesting it might possess lighter side effects (Fig. S1). Also, the dose of $10 \mathrm{mg} / \mathrm{kg}$ olaparib was chosen for further investigation as a result of the highest survival rate among all olaparib groups.

\section{Olaparib relieved IAV-induced lung injury in mice}

As olaparib treatment evidently reduced the virus-induced mortality, pathological changes were further explored to assess the influences of olaparib on the lung injury severity. In comparison with the control group, the lung index of mice in the IAV group was significantly higher than that in the control group, and olaparib treatment remarkably reduced the lung index, suggesting that olaparib could alleviate the pulmonary edema induced by IVA infection (Fig. 2a). As shown in Fig. $2 b$, higher mRNA expression of IAV was detected in IAV lungs, while no virus was detected in the control lung tissues, indicating the direct relationship between IAV infection and pathological manifestations of mice model. Unsurprisingly, less IAV was detected in the lungs of olaparib-treated IAV group, indicating the antiviral effect of this drug functions in the IAV model mice. Virus infection always correlated with leukocyte filtration to the target organs. We next determined the MPO levels and the marker of leucocytes, in lung tissues. Figure $2 \mathrm{c}$ showed that IAV infection elevated the MPO levels compared with that in the control group, whereas olaparib evidently reduced MPO levels, illustrating that olaparib reduced leucocyte infiltration to the lungs of IAV mice. Morphologic analysis was performed using H\&E staining of lung tissues. As shown in Fig. 2d, extensive inflammatory cell infiltration, especially around bronchioles, was presented in IAV lungs, signifying lung edema might lead to the breathing difficulty in infected mice. And olaparib treatment attenuated the pathological abnormalities in IAV lungs. Lung histopathological grading numerically pointed that olaparib rectified the lung injury caused by IAV infection (Fig. 2e).

\section{Olaparib reduced IAV-induced cytokine release in murine lungs}

Virus-infected pneumonia always comes along with abnormal release of inflammatory cytokines. Next the level of inflammatory cytokines in lung homogenate was detected to investigate whether olaparib could influence the release of inflammatory cytokines in lung tissue. As shown in Fig. 3a, b, c, d, e, compared with those in the control group, pro-inflammatory cytokines, IL- $1 \beta$, TNF- $\alpha$, IFN- $\gamma$, IL- 6 , and IL-4, were significantly elevated, and anti-inflammatory cytokine, IL-10, was remarkably descended in the IAV group, while olaparib treatment obviously rectified the abnormal release of the above inflammatory cytokines, meaning that olaparib might possess anti-inflammatory effect in murine lung tissue under the IAV context.
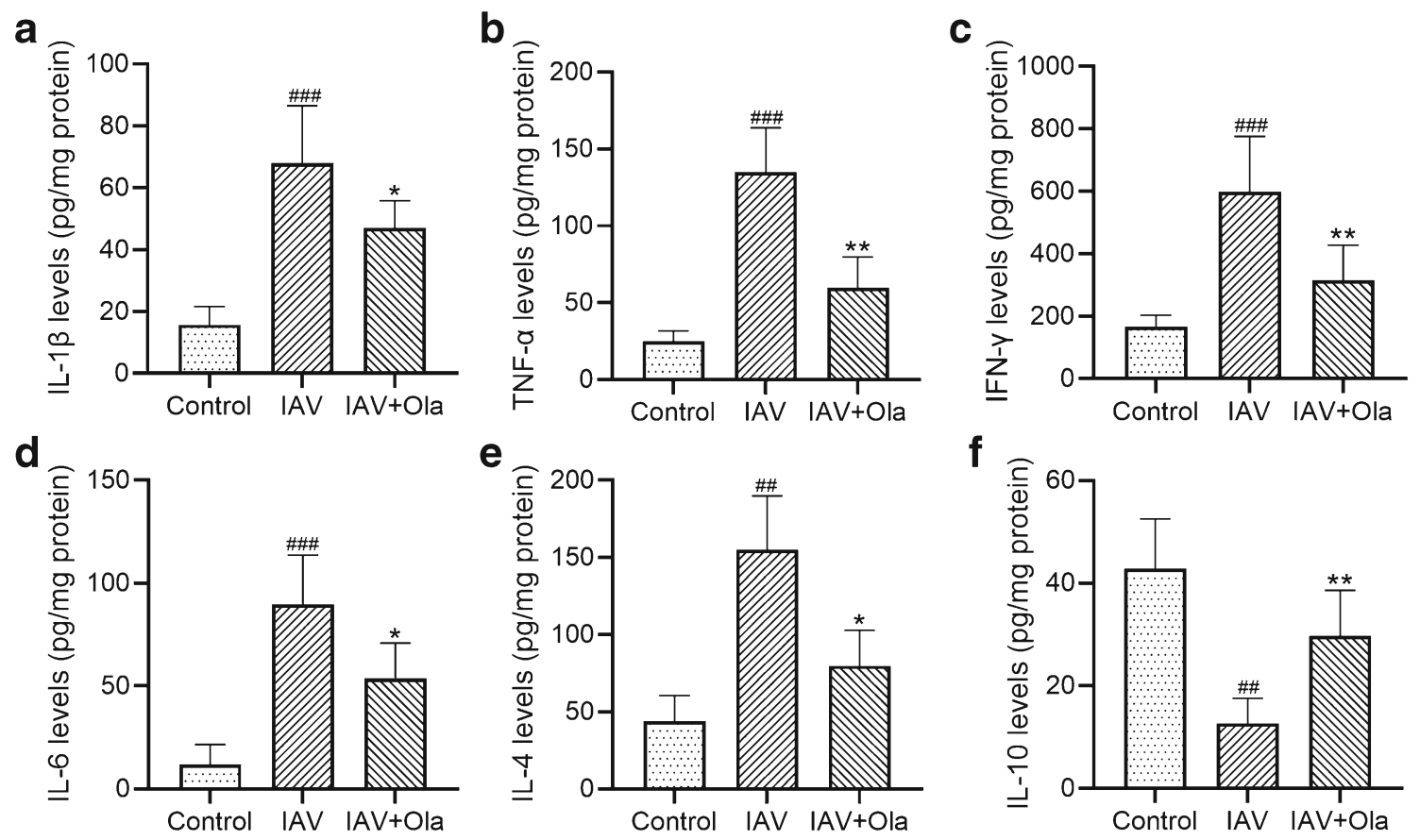

Fig. 3 Effects of olaparib on influenza virus challenge-induced cytokine release in lung homogenate at day 6 post-infection ( $n=8$ for each group). IL-1 $\beta$ (a), TNF- $\alpha$ (b), IFN- $\gamma$ (c), IL-6 (d), IL-4 (e), and IL-10 (f) were

measured by ELISA. Data were presented as mean $\pm \mathrm{SD} .{ }^{\# \#} p<0.01$ and ${ }^{\# \# \#} p<0.001$ compared with the control. ${ }^{*} p<0.05$ and ${ }^{* *} p<0.01$ compared with IAV 
a

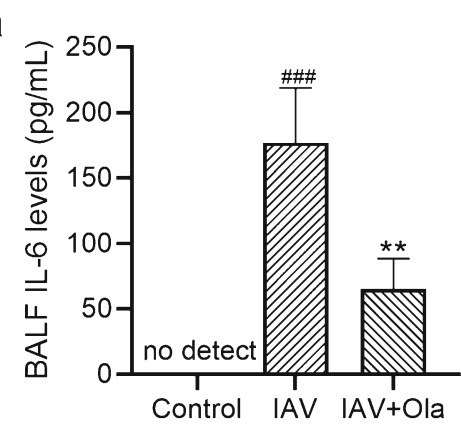

d

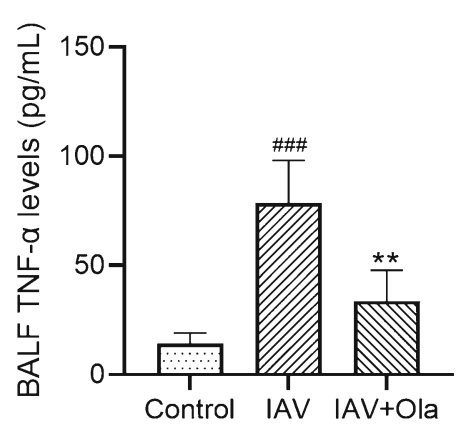

b

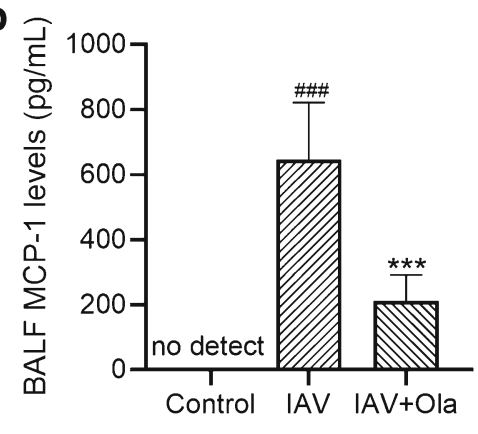

e

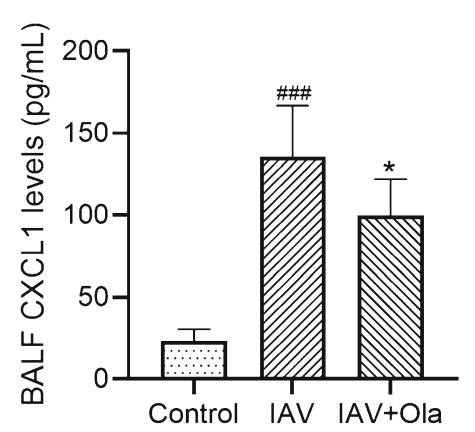

C

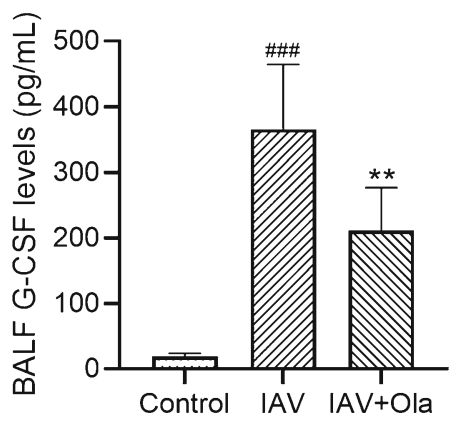

f

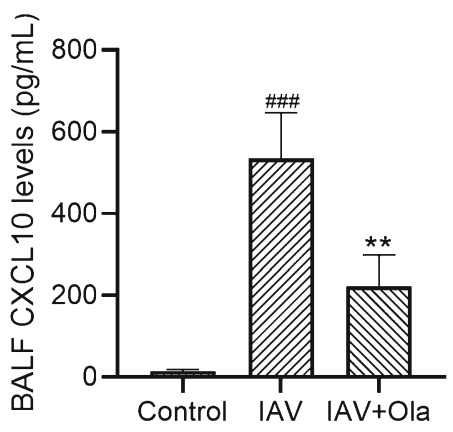

g

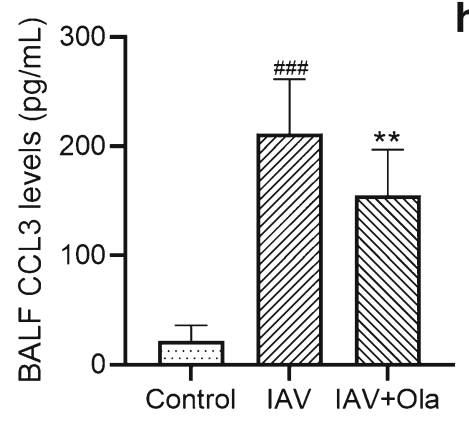

h

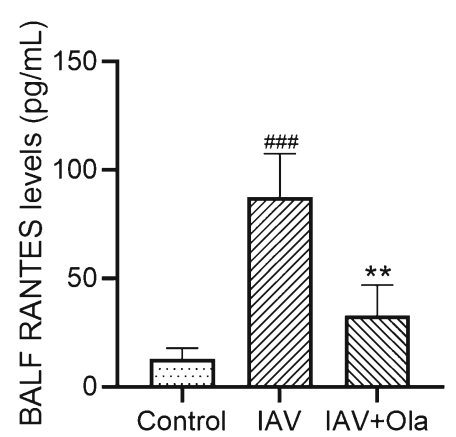

Fig. 4 Effects of olaparib on influenza virus challenge-induced cytokine and chemokine release in the bronchoalveolar lavage fluid (BALF) at day 6 post-infection ( $n=8$ for each group). IL-6 (a), MCP-1 (b), G-CSF (c), TNF- $\alpha$ (d), CXCL1 (e), CXCL10 (f), CCL3 (g), and RANTES (h) were

measured by ELISA. Data were presented as mean \pm SD. ${ }^{\# \# \#} p<0.001$ compared with the control. ${ }^{*} p<0.05,{ }^{* *} p<0.01$, and ${ }^{* * *} p<0.001$ compared with IAV

\section{Olaparib reduced IAV-induced cytokine release in murine BALF}

The detection of cytokine/chemokine in BALF samples at day 6 post-infection showed that IL-6, MCP-1, G-CSF, TNF- $\alpha$, CXCL1, CXCL10, CCL3, and RANTES were remarkably increased in the IAV group compared with those in the control group, while olaparib treatment significantly reduced the abnormal increased levels of the above cytokine/chemokines, which was similar with the results obtained from lung tissue (Fig. 4a-h).

\section{Olaparib decreased PARylated protein content and p65, IKBa phosphorylation in IAV-challenged lung tissues}

To explore the mechanisms underlying the protective effect of olaparib against IAV-induced injury to murine lungs, western blot was performed to detect the PARPs, the marker of apoptosis. IAV infection increased the PARylated protein content in lung homogenate samples compared with the control group, while PARP inhibitor olaparib significantly reduced the PARylated protein content in IAV-infected samples (Fig. 5a and c). NF-KB genes are reported to be the targets of olaparib in the context of LPS stimulation to the lungs [12]. As shown in Fig. 5b, d, and e, IAV remarkably elevated the p-p65 and $\mathrm{p}-\mathrm{I} \kappa \mathrm{B} \alpha$ protein expression in lung homogenates compared with those of the control group, while olaparib obviously decreased the abnormal increase of the two proteins, suggesting that olaparib inhibits the activation of NF- $\mathrm{BB}$ signaling pathway in the condition of IAV infection. And there were no significant differences of the total expressions of p65 and IkBa among different groups (Fig. 5b). 


\section{Discussion}

The highly transmissible human pathogen IAV mainly attacks human respiratory epithelium with its hemagglutinin binding with sialic acid to initiate endocytosis [18]. Severe symptoms after IAV infection are commonly found in the infant, elderly, pregnant, as well as the immunocompromised populations [19-22]. IAV can cause serious pandemics in a short time, with shocking and heart-wrenching facts in history, e.g., the 1918 Great Influenza killed almost 1/30 of the global population only in 1.5 years. Also IAV is capable of generating new strains adapted to human beings. That is the very reason that influenza vaccines have to be updated regularly. Also antiviral drugs have to be replaced as a result of the increasing drug resistance developed by viable IAV subtypes. Thus, it is urgent to explore novel anti-IAV drugs to enrich the available antiviral drug bank. This study investigated for the first time the possible role of olaparib on IAV infection in a murine model and found that PARP-1 inhibitor olaparib remarkably relieved IAV-induced lung injury and lung inflammation possibly via inhibition of PARP, as well as $\mathrm{p} 65$, and $\mathrm{I} \kappa \mathrm{B} \alpha$ phosphorylation.

PARP has been indicated to be the target of treatment strategy against cancers and inflammatory disorders [23, 24]. The pro-inflammatory effect of PARP-1 has been highlighted in a variety of non-pulmonary and pulmonary inflammatory diseases, including arthritis, allergic encephalomyelitis, asthma, acute lung injury (ALI), and so on [25-27]. PARP-1 is activated and involved in the lungs of allergen-induced asthma animal models via modulating immune cell recruitment, airway modeling, as well as cytokine production, mainly $\mathrm{Th} 2$ cytokines [27-29]. PARP-1 is reported to play a critical role in LPS-induced and mechanical ventilation-induced ALI in mice $[30,31]$. Hence PARP-1 might be a convincing target for the prevention and treatment of lung inflammatory injury.

Genetic as well as pharmacological measures modulating PARP-1 activity were proved to be effective to alleviate the lung inflammation and injury experimentally from the aspects of reducing neutrophil infiltration and macrophage accumulation [32], blocking the activation of NF-KB and AP-1 [33]. Additionally, PARP-1 inhibition can relieve the secondary kidney injury induced by ALI [34]. As a potent PARP inhibitor, olaparib has already been approved to be used in cancer patients for clinical trials with acceptable toxicity. Olaparib downregulates NF-kB-related genes including TNF- $\alpha$ and IL-1 $\beta$, decreases neutrophil, and alleviates edema of LPSstimulated murine lungs [12]. Our study found that olaparib possesses protective effects against IAV-induced lung inflammation, suggesting that this drug might exert non-specific anti-inflammatory roles. As the outbreak of severe coronavirus infection since December 2019, it is urgent to explore the mechanisms of virus-induced damage to lung tissues or other organs, as well as develop more effective antiviral drugs or therapies to prevent related damages to patients. Our study
Fig. 5 Effects of olaparib on influenza virus challenge-induced PARylated protein content and $\mathrm{p} 65, \operatorname{IkB} \alpha$ phosphorylation in lung tissues at day 6 postinfection ( $n=8$ for each group). Western blot analysis of PARylated protein content (a) and p-p65, p65, p- I $\kappa B \alpha$, and I $\mathrm{B} \alpha$ (b) in lung samples from each experimental group. The relative expressions were normalized to control (c, d, e). Data are presented as mean $\pm \mathrm{SD}$. ${ }^{\# \#} p<0.01$ and $^{\# \# \# p<0.001}$ compared with the control. ${ }^{* * *} p<0.01$ and ${ }^{* * * *} p<0.001$ compared with IAV a
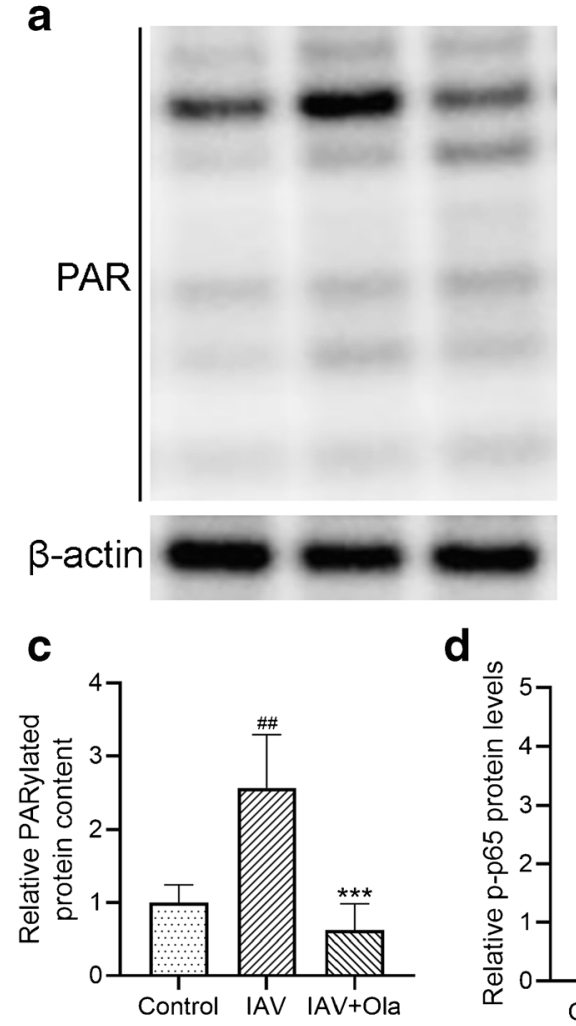

b

d

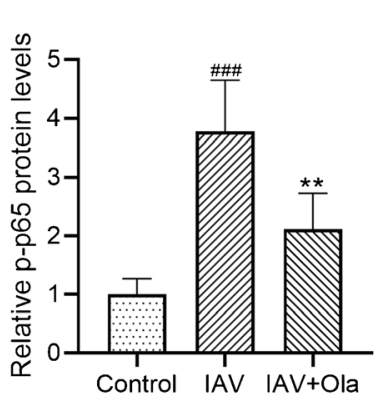

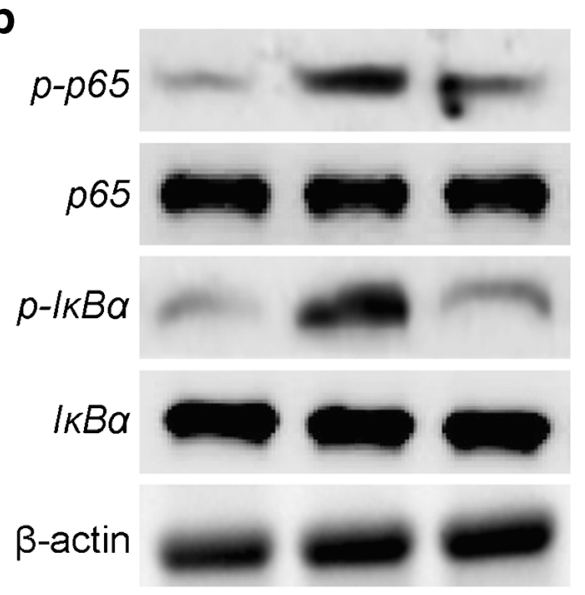

e

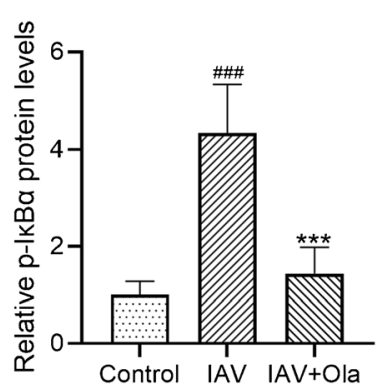


explored the protective effects of olaparib in experimental virus-induced pneumonia, suggesting its possible application in viral pneumonia treatment in the future.

There are some shortcomings in our study. We did not perform toxicity analysis about the influence of olaparib on normal physiology of mice. The relationship between PARP$1 / \mathrm{NF}-\mathrm{KB}$ and IAV-induced lung inflammation as well as the concrete mechanisms was not well illustrated. Related experiments would be performed in the future study.

\section{Conclusions}

The present study successfully constructed the pneumonia murine model using IAV. Olaparib decreased IAV-induced mortality in mice, lung injury, and cytokine production, possibly via modulation of PARP-1/NF-KB axis. This study indicates the non-specific anti-inflammatory effect of olaparib in lung disorders and would shine light on the development for treatment against pneumonia infected by viable IAV subtypes.

\section{Compliance with ethical standards}

Conflict of interest The authors declare that they have no competing interests.

Ethical approval All the animal-involved experiments were performed in accordance with the Animal Care and Use Committee of Cangzhou Central Hospital.

Informed consent Not applicable.

\section{References}

1. Rambaut A, Pybus OG, Nelson MI, Viboud C, Taubenberger JK, Holmes EC (2008) The genomic and epidemiological dynamics of human influenza A virus. Nature 453(7195):615-U612

2. Bedford T, Riley S, Barr IG, Broor S, Chadha M, Cox NJ, Daniels RS, Gunasekaran CP, Hurt AC, Kelso A, Klimov A, Lewis NS, Li XY, McCauley JW, Odagiri T, Potdar V, Rambaut A, Shu YL, Skepner E, Smith DJ, Suchard MA, Tashiro M, Wang DY, Xu XY, Lemey P, Russell CA (2015) Global circulation patterns of seasonal influenza viruses vary with antigenic drift. Nature 523(7559):217-U206

3. Chaiyakunapruk N, Kotirum S, Newall AT, Lambach P, Hutubessy RCW (2018) Rationale and opportunities in estimating the economic burden of seasonal influenza across countries using a standardized WHO tool and manual. Influenza Other Respir Viruses 12(1): 13-21

4. Belongia EA, Simpson MD, King JP, Sundaram ME, Kelley NS, Osterholm MT, McLean HQ (2016) Variable influenza vaccine effectiveness by subtype: a systematic review and meta-analysis of test-negative design studies. Lancet Infect Dis 16(8):942-951

5. Beck C, Robert I, Reina-San-Martin B, Schreiber V, Dantzer F (2014) Poly (ADP-ribose) polymerases in double-strand break repair: focus on PARP1, PARP2 and PARP3. Exp Cell Res 329(1): $18-25$
6. Masutani M, Nozaki T, Wakabayashi K, Sugimura T (1995) Role of poly (ADP-ribose) polymerase in cell-cycle checkpoint mechanisms following gamma-irradiation. Biochimie 77(6):462-465

7. Hassa PO, Hottiger MO (2002) The functional role of poly (ADPribose) polymerase 1 as novel coactivator of NF-kappaB in inflammatory disorders. Cell Mol Life Sci 59(9):1534-1553

8. Liaudet L, Pacher P, Mabley JG, Virag L, Soriano FG, Hasko G, Szabo C (2002) Activation of poly(ADP-ribose) polymerase-1 is a central mechanism of lipopolysaccharide-induced acute lung inflammation. Am J Respir Crit Care Med 165(3):372-377

9. Dharwal V, Naura AS (2018) PARP-1 inhibition ameliorates elastase induced lung inflammation and emphysema in mice. Biochem Pharmacol 150:24-34

10. Michaelis M, Geiler J, Klassert D, Doerr HW, Cinatl J Jr (2009) Infection of human retinal pigment epithelial cells with influenza A viruses. Invest Ophthalmol Vis Sci 50(11):5419-5425

11. Liang X, Huang Y, Pan X, Hao Y, Chen X, Jiang H, Li J, Zhou B, Yang Z (2020) Erucic acid from Isatis indigotica Fort. suppresses influenza $A$ virus replication and inflammation in vitro and in vivo through modulation of NF-kappaB and p38 MAPK pathway. J Pharm Anal 10(2):130-146

12. Kapoor K, Singla E, Sahu B, Naura AS (2015) PARP inhibitor, olaparib ameliorates acute lung and kidney injury upon intratracheal administration of LPS in mice. Mol Cell Biochem 400(1-2): 153-162

13. Hatayama K, Nosaka N, Yamada M, Yashiro M, Fujii Y, Tsukahara H, Liu K, Nishibori M, Matsukawa A, Morishima T (2019) Combined effect of anti-high-mobility group box-1 monoclonal antibody and peramivir against influenza A virus-induced pneumonia in mice. J Med Virol 91(3):361-369

14. Belperio JA, Keane MP, Burdick MD, Londhe V, Xue YY, Li K, Phillips RJ, Strieter RM (2002) Critical role for CXCR2 and CXCR2 ligands during the pathogenesis of ventilator-induced lung injury. J Clin Invest 110(11):1703-1716

15. Yashiro M, Tsukahara H, Matsukawa A, Yamada M, Fujii Y, Nagaoka Y, Tsuge M, Yamashita N, Ito T, Yamada M, Masutani H, Yodoi J, Morishima T (2013) Redox-active protein thioredoxin1 administration ameliorates influenza A virus (H1N1)-induced acute lung injury in mice. Crit Care Med 41(1):171-181

16. Pulli B, Ali M, Forghani R, Schob S, Hsieh KL, Wojtkiewicz G, Linnoila JJ, Chen JW (2013) Measuring myeloperoxidase activity in biological samples. PLoS One 8(7):e67976

17. Li YC, Peng SZ, Chen HM, Zhang FX, Xu PP, Xie JH, He JJ, Chen JN, Lai XP, Su ZR (2012) Oral administration of patchouli alcohol isolated from Pogostemonis Herba augments protection against influenza viral infection in mice. Int Immunopharmacol 12(1):294-301

18. Hutchinson EC (2018) Influenza Virus. Trends Microbiol 26(9): 809-810

19. Vazquez-Perez JA, De La Rosa-Zamboni D, Vega-Sanchez AE, Gutierrez-Gonzalez LH, Tellez-Navarrete NA, Campos F, Guadarrama-Perez C, Sandoval JL, Castillejos-Lopez M, Jimenez-Juarez RN, Sanchez-Huerta JL, Perez-Mendez BB, Perez-Padilla R (2019) Amino acid changes in HA and determinants of pathogenicity associated with influenza virus A H1N1pdm09 during the winter seasons 2015-2016 and 20162017 in Mexico. Virus Res 272:197731

20. Destras G, Pichon M, Simon B, Valette M, Escuret V, Bolze PA, Dubernard G, Gaucherand P, Lina B, Josset L (2019) Impact of pregnancy on intra-host genetic diversity of influenza A viruses in hospitalised women: a retrospective cohort study. J Clin Med 8(11): 1974

21. Veiga A, Martins LG, Riediger I, Mazetto A, Debur MDC, Gregianini TS (2020) More than just a common cold: endemic coronaviruses OC43, HKU1, NL63, and 229E associated with severe acute respiratory infection and fatality cases among healthy adults. J Med Virol. https://doi.org/10.1002/jmv.26362 
22. Han J, Perez J, Schafer A, Cheng H, Peet N, Rong L, Manicassamy B (2018) Influenza virus: small molecule therapeutics and mechanisms of antiviral resistance. Curr Med Chem 25(38):5115-5127

23. Killock D (2017) Targeted therapy: ARIEL3 - broad benefit of PARP inhibitors in ovarian cancer. Nat Rev Clin Oncol 14(12):713

24. Lord CJ, Ashworth A (2008) Targeted therapy for cancer using PARP inhibitors. Curr Opin Pharmacol 8(4):363-369

25. Ba XQ, Garg NJ (2011) Signaling mechanism of poly (ADPribose) polymerase-1 (PARP-1) in inflammatory diseases. Am J Pathol 178(3):946-955

26. Rosado MM, Bennici E, Novelli F, Pioli C (2013) Beyond DNA repair, the immunological role of PARP-1 and its siblings. Immunology 139(4):428-437

27. Sethi GS, Dharwal V, Naura AS (2017) Poly (ADP-ribose) polymerase-1 in lung inflammatory disorders: a review. Front Immunol 8:1172

28. Boulares AH, Zoltoski AJ, Sherif ZA, Jolly P, Massaro D, Smulson ME (2003) Gene knockout or pharmacological inhibition of poly (ADP-ribose) polymerase-1 prevents lung inflammation in a murine model of asthma. Am J Resp Cell Mol 28(3):322-329

29. Suzuki Y, Masini E, Mazzocca C, Cuzzocrea S, Ciampa A, Suzuki $\mathrm{H}$, Bani D (2004) Inhibition of poly (ADP-ribose) polymerase prevents allergen-induced asthma-like reaction in sensitized Guinea pigs. J Pharmacol Exp Ther 311(3):1241-1248

30. Liu L, Ke Y, Jiang X, He F, Pan L, Xu L, Zeng X, Ba X (2012) Lipopolysaccharide activates ERK-PARP-1-RelA pathway and promotes nuclear factor-kappaB transcription in murine macrophages. Hum Immunol 73(5):439-447

31. Vaschetto R, Kuiper JW, Chiang SR, Haitsma JJ, Juco JW, Uhlig S, Plotz FB, Della Corte F, Zhang H, Slutsky AS (2008) Inhibition of poly (adenosine diphosphate-ribose) polymerase attenuates ventilator-induced lung injury. Anesthesiology 108(2):261-268

32. Zerfaoui M, Naura AS, Errami Y, Hans CP, Rezk BM, Park J, Elsegeiny W, Kim H, Lord K, Kim JG, Boulares AH (2009) Effects of PARP-1 deficiency on airway inflammatory cell recruitment in response to LPS or TNF: differential effects on CXCR2 ligands and Duffy antigen receptor for chemokines. J Leukoc Biol 86(6):1385-1392

33. Wang G, Huang X, Li Y, Guo K, Ning P, Zhang Y (2013) PARP-1 inhibitor, DPQ, attenuates LPS-induced acute lung injury through inhibiting NF-kappaB-mediated inflammatory response. PLoS One 8(11):e79757

34. Si MKH, Mitaka C, Tulafu M, Abe S, Kitagawa M, Ikeda S, Eishi Y, Kurata S, Tomita M (2013) Inhibition of poly (adenosine diphosphate-ribose) polymerase attenuates lung-kidney crosstalk induced by intratracheal lipopolysaccharide instillation in rats. Respir Res 14:126

Publisher's note Springer Nature remains neutral with regard to jurisdictional claims in published maps and institutional affiliations. 